\title{
Relative bioavailability of diazoxide, manufactured at two different international locations, in healthy participants under fasting conditions: an open-label, two- stage, adaptive, sequential two-period crossover study
}

\author{
Martin Otto Behm ${ }^{1,2^{*}}$, Jialin Xu', Deborah Panebianco ${ }^{1}$ and Paul Fackler ${ }^{1}$
}

\begin{abstract}
Theoretically, calculating the sample size required for achieving the desired power is straightforward with true coefficient of variance (CV) estimates and true test/reference geometric mean ratios (GMRs) of selected parameters. However, true variance estimates are often not known and are, instead, based on published literature or pilot trials. An open-label, two-stage, adaptive, sequential two-period crossover study was used to determine relative bioavailability of a single $100 \mathrm{mg}$ dose of diazoxide manufactured at two different international sites. This design was utilized due to the lack of intra-subject variance estimation data for diazoxide pharmacokinetic parameters. Second-stage sample size was estimated using intra-participant variance from the first stage. Thirty-six healthy adults (age, 18-55 years) were randomized (1:1) to receive a single dose of test/reference product, a single dose of diazoxide (100 mg capsule), under fasting conditions with crossover after 14 days. GMRs (90\% confidence interval $[\mathrm{Cl}])$ of area under curve from time zero to infinity $\left(A \cup C_{0 \text {-inf }}\right)$ and maximum plasma concentrations $\left(C_{\max }\right)$ of test versus reference product were $1.06(1.03-1.10)$ and $1.17(1.14-1.20)$, respectively. Bioequivalence was declared at stage 1 because $90 \%$ Cls were between 0.80 and 1.25; stage 2 was not initiated since the estimated power was $>99 \%$ for both $A \cup C_{0 \text {-inf }}$ and $C_{\max }$. Results of this study demonstrate efficient use of an adaptive, two-stage sequential design to assess bioequivalence. Similar study design may be effectively used in other bioequivalence studies wherein CV and GMRs of relevant parameters are unknown and sample size estimation is difficult.
\end{abstract}

Keywords: Adaptive design, Bioavailability, Bioequivalence, Diazoxide, Two-stage sequential design

\section{Background}

Pharmacokinetic studies are most preferred for assessing the bioequivalence of two drug formulations (FDA 2013). Endpoints such as peak plasma concentration $\left(\mathrm{C}_{\max }\right)$ and area under the plasma concentration time curve (AUC), which reflect rate and extent of absorption, respectively, are commonly used parameters. The $90 \%$ confidence interval $(\mathrm{CI})(\alpha$, type I error $=0.05)$ for

\footnotetext{
* Correspondence: martin_behm@merck.com

${ }^{1}$ Merck and Co., Inc., Kenilworth, NJ, USA

2351 N Sumneytown Pike, North Wales, PA 19454, USA
}

the geometric mean ratio (GMR) of test/reference is derived, and generally the two formulations are declared bioequivalent when the $90 \% \mathrm{CIs}$ for $\mathrm{C}_{\max }$ and $\mathrm{AUC}$ fall within the limits of 0.80 and 1.25 (Davit et al. 2008, Fuglsang 2013). Health Canada specifically requires that $90 \% \mathrm{CI}$ for $\mathrm{AUC}$ and the point estimate of $\mathrm{C}_{\max }$ fall within the limits of 0.80 and 1.25 to declare bioequivalence (Health Canada 2012).

Sample size estimation is a vital step for bioequivalence studies comparing a reference product with a new/ generic (test) product. To estimate sample size, estimates of intra-subject variance and effect size are needed to 
achieve the desired power. As bioequivalence studies are commonly conducted using crossover study designs, variance estimates are based on a withinparticipant coefficient of variation $(\mathrm{CV})$, and effect size is determined using GMRs of selected parameters for the two formulations. Although a minimum sample size is obtained at perfect equivalence (assumed $G M R=1.00$ ), some departure from perfect equivalence is commonly assumed while estimating sample size (Fenta 2014, Potvin et al. 2008).

Theoretically, calculating the sample size required for achieving the desired power is straightforward with true $\mathrm{CV}$ estimates and true test/reference GMRs of selected parameters. However, true variance estimates are not known and are, instead, based on published literature or pilot trials (Fuglsang 2013). When variance estimates are too low or choice of effect size is overly optimistic, the study can be underpowered. In contrast, when variance estimates are too high, unnecessarily large sample sizes can result.

For formulations intended for systemic availability, the Food and Drug Administration (FDA) recommends using a two-period, two-sequence, two-treatment, single-dose crossover study using healthy participants (FDA 2013). Approaches to the design of bioequivalence studies involving highly variable drugs have been described (O'Brien and Fleming 1979, Pocock 1982, Potvin et al. 2008). With scarce information on CV estimates, an adaptive design, in which the second-stage sample size is based on the estimated intra-participant variance from the first stage, is an approach similar to a sequential design. Method $\mathrm{C}$ described by Potvin et al. and validated via a series of simulated bioequivalence studies (Potvin et al. 2008) is the adaptive design recommended by Health Canada (Health Canada 2012).

Potvin's method $\mathrm{C}$ uses an adaptive, two-stage sequential design, wherein, stage 2 is not initiated if power is $\geq 80 \%(\alpha$, type I error $=0.05)$ at the end of stage 1 (interim analysis). If bioequivalence criteria are met (or not met), the formulations are considered bioequivalent (or not bioequivalent) at this stage. When power is $80 \%$ at the end of stage 1, calculation of the required sample size is based on within-participant variability of stage 1 and an assumed GMR (Polli et al. 2012).

Diazoxide, a widely used potassium channel activator and hyperglycemic agent which inhibits insulin release from the pancreas and exerts an extrapancreatic effect (Gribble et al. 1997), has been in use for more than 40 years as a hyperglycemic agent in the management of hypoglycemia due to hyperinsulinism (PROGLYCEM Product Monograph 2015). Diazoxide can also be used as an antihypertensive agent in emergency situations such as acute and malignant hypertension (Dayton et al. 1975, Vaziri 1984). The plasma half-life following intravenous administration is $28 \pm 8.3$ hours (PROGLYCEM Product Monograph 2015).

Several oral prescription formulations of diazoxide, including PROGLYCEM ${ }^{\circ} 100$ mg capsules (Merck Canada Inc., Canada, [manufactured in Italy]) are available. Recently, production of PROGLICEM ${ }^{\circ} 100 \mathrm{mg}$ hard capsules, (MSD Sharp \& Dohme GmbH, Germany) was initiated at a facility in France. Same excipients and manufacturing procedures were used at both manufacturing sites. The $\mathrm{f} 2$ values of the two dissolution profiles from Italy and Germany lots were 69, 78, and 76 at $\mathrm{pH}$ $1.2,4.5$, and 6.8 , respectively, using $2 \%$ sodium dodecyl sulfate as dissolution media. Thus the dissolution profiles of capsules produced at both sites were similar as indicated by the f2 similarity test. The US FDA Center for Drug Evaluation and Research recommends that an in vivo bioequivalence study be conducted to support regulatory approval of certain major changes in components, composition, and manufacturing of an approved formulation (FDA 2010). Therefore, we conducted a bioequivalence study to determine the in vivo relative bioavailability of a single dose of diazoxide (100 mg) manufactured in either France (test product) or Italy (reference product). The intra-participant variability estimates of $\mathrm{C}_{\max }$ and AUC for oral diazoxide are not available in the published literature or from accessible unpublished studies; thus, an adaptive, two-stage sequential design (Potvin's method $\mathrm{C}$ ) was used for sample size and power estimation.

\section{Methods/experimental}

The study was approved by the Ethics Review Board, Optimum Clinical Research Inc. and was conducted in accordance with Good Clinical Practice guidelines, Current Therapeutic Products Directorate (Canada) guidance documents, and the 2008 Declaration of Helsinki. All participants signed a written consent form (Privacy Consent Form and Volunteer Consent Form) before enrollment in the study.

\section{Study design}

This open-label, single-dose, randomized, two-period, two-treatment, two-sequence, two-stage crossover study (protocol number: MK-6783-003-01) conducted in healthy adults tested the hypothesis that a single dose of diazoxide (100 mg capsule) manufactured in Patheon, France (test product) is bioequivalent to a single dose of diazoxide (100 mg capsule) manufactured in Comazzo, Italy (reference product). Participants underwent routine screening and check-in tests by Pharma Medica Research Inc. before in-house confinement for pharmacokinetic evaluations at stage 1 . At completion of stage 1 , an interim analysis was planned to estimate the power and test 
bioequivalence if adequate $(\geq 90 \%)$ power has been achieved before initiating stage 2 .

\section{Participants}

Healthy, nonsmoking men and women, aged 18 to 55 years, with body mass index $\geq 18.5$ and $\leq 30.0 \mathrm{~kg} / \mathrm{m}^{2}$ and willing to use an acceptable, effective method of contraception were included in the study. Exclusion criteria included clinically significant findings in vital sign measurements, physical examinations,12-lead electrocardiograms, or laboratory assessments; specific diseases based on medical history, physical examinations, and laboratory assessments; history of drug or alcohol addiction requiring treatment; positive urine test results for drugs of abuse or urine cotinine; difficulty fasting or consuming standard meals; intolerance of venipuncture; use of tobacco or nicotine-containing products within 6 months of drug administration; special diet within 30 days of drug administration; participation in another clinical trial or receiving an investigational product within 30 days of drug administration; donated or lost whole blood (including for clinical trials) $\geq 50 \mathrm{~mL}$ and $\leq 499 \mathrm{~mL}$ within 30 days of drug administration or $\geq 500 \mathrm{~mL}$ within 56 days of drug administration; tattoo or body piercing within 30 days of drug administration; and known history or presence of hypersensitivity or idiosyncratic reaction to diazoxide, thiazide, or any other drug substances with similar activity. Women who were pregnant (based on serum human chorionic gonadotropin levels) or lactating, had used oral or transdermal hormonal contraceptives within 21 days of drug administration, or had used implanted, injected, intravaginal, or intrauterine hormonal contraceptives within 6 months of drug administration were excluded. Mentally or legally incapacitated participants or those with significant emotional problems at the time of screening or expected during the conduct of the study or had a history of a clinically significant psychiatric disorder over the preceding 5 years were excluded.

\section{Interventions and participant evaluations}

In stage 1, eligible participants were randomized to receive one treatment sequence (treatment sequences test/reference and reference/test to maintain a 1:1 ratio) according to a predetermined, computer-generated randomization scheme (procedure PLAN in SAS ${ }^{\circ}$ ). Participants were confined in-house for at least 10 hours before and 24 hours after drug administration. Participants received a single dose of test product (diazoxide, $100 \mathrm{mg}$ hard capsule [PROGLICEM ${ }^{\ominus} 100$ mg Hard Capsules; distributor, MSD Sharp \& Dohme GmbH, Germany; manufacturer, Patheon, France; Batch No.: 3107A]) or reference product (diazoxide, $100 \mathrm{mg}$ capsule, [PROGLYCEM ${ }^{\circ} 100$ mg capsules; distributor, Merck Canada Inc., Canada; manufacturer, Comazzo, Italy; Lot No.: 120118]) per randomization, on January 24, 2014 (period 1). After a washout period of at least 14 days ( \pm 3 hours), participants received the other study treatment on February 07, 2014 (period 2).

Diazoxide capsules were administered with $240 \mathrm{~mL}$ $( \pm 5 \mathrm{~mL})$ of room temperature, potable water after at least 10 hours of overnight fasting. A hand and mouth check-up was performed immediately after drug administration to ensure study drug was swallowed. Participants remained seated for 4 hours after drug administration, and a standard protocol for requirement of ambulation with postural changes was instituted.

After fasting for at least 4 hours after drug administration during each study period, participants were provided with standardized xanthine-free meals with caffeine-free beverages. Other standardized identical meals were served throughout the confinement periods. Water consumption was restricted for 1 hour before and after drug administration. Participants were monitored for adverse events (AEs) during the confinement period. They were contacted by phone approximately 14 days after drug administration for assessment of AEs after confinement. Provisions were made for additional health monitoring procedures during the study period if considered necessary by investigators. Vital signs were measured before dosing and at 2 and 8 hours $( \pm 20$ minutes $)$ after dosing. A finger-stick glucose test was performed within 60 minutes before dosing and at $2,4,6$, and 8 hours $( \pm 20$ minutes) after dosing. Participants with predose capillary glucose values $<3.6 \mathrm{mmol} / \mathrm{L}$ were evaluated by investigators before dosing. Clinical laboratory tests for hematology, biochemistry, and urinalysis were conducted at the end of the study.

\section{Collection and processing of samples}

During each study period, blood samples were collected by direct venipuncture or from an indwelling cannula before dosing and over a 72-hour period after dosing (at hourly intervals for 12 hours, and then at 14, 18, 24, 36, 48,60 , and 72 hours) into prechilled, labeled blood collection tubes containing K2-ethylenediaminetetraacetic acid as the anticoagulant. During the confinement period, samples were collected at participants' homes. Subsequently, participants were required to visit the clinic for collection of remaining samples. Approximately $170 \mathrm{~mL}$ of blood was collected from each participant over the entire study for pharmacokinetic analysis.

After collection, samples were maintained in an ice bath until processing. Samples were centrifuged at approximately $4^{\circ} \mathrm{C}$ for approximately 10 minutes at 3,000 rpm within 30 minutes of collection and then stored in an ice bath. Plasma was withdrawn, divided into two approximately equal aliquots, placed in labeled polypropylene 
screw-top tubes, and stored at $-25 \pm 10^{\circ} \mathrm{C}$ within 60 minutes of collection.

\section{Pharmacokinetic outcomes}

Plasma samples were assayed for diazoxide using a validated analytical method, according to the principles of good laboratory practice (GLP) [US Department of Health and Human Services 2010, Organisation for Economic Co-operation and Development (OECD) 2006). Tandem mass spectrometry was used for diazoxide detection. This analytical method was developed and validated at Pharma Medica Research Inc. All samples from a given subject were analyzed in a single analytical batch. The lower limit of quantitation (LLOQ) of this assay was $50.0 \mathrm{ng} / \mathrm{mL}$ and the precision and accuracy at this level during study sample analysis were $5.6 \%$ and $100.2 \%$ respectively. Any value below the LLOQ is reported as below the limit of quantitation (BLQ).

Pharmacokinetic parameters included $\mathrm{C}_{\max }$, AUC from time zero to time of the last measurable analyte concentration $\left(\mathrm{AUC}_{0-\mathrm{t}}\right)$, AUC from time zero to infinity $\left(\mathrm{AUC}_{0 \text {-inf }}\right)$, time to $\mathrm{C}_{\max }\left(\mathrm{T}_{\max }\right)$, apparent firstorder elimination rate constant $\left(\mathrm{K}_{\mathrm{el}}\right)$, and apparent elimination half-life $\left(t_{1 / 2}\right)$.

\section{Safety measures}

Safety assessment was the frequency and severity of AEs (medical occurrences during the study period after the first dose of study drug was administered) in the safety cohort, which included participants who received at least one dose of study drug. Non-treatment-emergent adverse events (NTEAEs) were defined as any AEs that occurred after a participant had signed the written consent form but before administration of the first dose of study drug.

\section{Two-stage adaptive design and sample size calculation} Based on the FDA (FDA 2013) and Health Canada (Health Canada 2012) recommendations, an adaptive two-stage design was used because within-participant $\mathrm{CV}$ estimates for diazoxide were not available. According to this method, the study was to be discontinued if power was $\geq 90 \%$ at stage 1 (interim analysis) regardless of bioequivalence test. The power was estimated using estimated CV from stage 1, assumed true GMR $=0.95$ and $\alpha=0.05$. However, if power was $<90 \%$, bioequivalence was to be evaluated again with $\alpha=0.0294$, and the study was to be discontinued if bioequivalence criteria were met. If bioequivalence criteria were not met, the sample size was to be recalculated for stage 2 using stage 1 results with $\alpha=0.0294$. Thereafter, a final bioequivalence evaluation was to be again attempted at stage 2 using pooled data from both stages with $\alpha=$ 0.0294 (Fig. 1). Bioequivalence criteria were based on log-transformed data: $90 \% \mathrm{CI}$ of the GMR of $\mathrm{AUC}_{0 \text {-inf }}$ must be between 0.80 and 1.25 and point estimate GMR of $C_{\max }$ must be between 0.80 and 1.25 in the interim analysis when power $\geq 90 \%$ (interim analysis when power $<90 \%$ or final analysis).

Planned average $\mathrm{N}$ varied by true $\mathrm{CV}$ and ranged from 36 (CV, 20\%) to 150 (CV, 55\%). This $\mathrm{N}$ range is similar to or less than that of a single-stage study design; however, a $\mathrm{CV}>25 \%$ represents high variability in bioequivalence studies. In stage 1 , planned $\mathrm{N}$ was 36 (cohort 1), which provided $>95 \%$ power for an assumed GMR of $95 \%$ if $C V$ was $\leq 20 \%$. For stage 2, planned total $\mathrm{N} \leq 150$ (cohorts 1 and 2) would maintain $\alpha$ below $5 \%$ and provide $\geq 70 \%$ power for $\mathrm{CV} \leq 55 \%$ (Fig. 1).

\section{Pharmacokinetic and statistical analyses}

The pharmacokinetic dataset comprised participants with estimates of $\mathrm{C}_{\max }$ and $\mathrm{AUC}$ parameters for two periods. The statistical dataset comprised participants from pharmacokinetic dataset but excluding identified outliers and/or predose concentrations $>5 \%$ of the corresponding $\mathrm{C}_{\max }$. The values considered as outliers should meet both of the following criteria 1) studentized residual value larger than 3 and 2) the value is outside the range determined by all other values for the same PK parameter including all formulations, as recommended by Health Canada (Health Canada 2012). The safety dataset included participants who received at least one dose of study drug.

$\mathrm{AUC}_{0 \text {-inf }}$ and $\mathrm{C}_{\max }$ values for each treatment were pooled, natural log-transformed, and analyzed in separate linear mixed-effects models with fixed-effects terms for treatment, period, and sequence. An unstructured covariance matrix was used to allow for unequal treatment variances and to model the correlation between different treatment measurements within the same participant via the repeated statement in $\mathrm{SAS}^{\circ}$ PROC MIXED. Kenward and Roger's method (Kenward and Roger 1997) was used to calculate the denominator degree of freedom for the fixed effects.

To address the study hypothesis, two-sided 90\% CIs for the true differences in means for log-transformed $\mathrm{AUC}_{0 \text {-inf }}$ and $\mathrm{C}_{\max }$ (test product-reference product) were calculated using the mean square error from the analysis of variance (ANOVA) and referencing a tdistribution. Confidence limits were exponentiated to obtain the $90 \%$ CIs for the $\mathrm{AUC}_{0 \text {-inf }}$ and $\mathrm{C}_{\max }$ true GMRs (test product-reference product).

Testing of sequence, period, and treatment effects were summarized using ANOVA. Estimates of inter-participant and intra-participant variances were also summarized. Interim analysis on $\mathrm{AUC}_{0 \text {-inf }}$ using the mixed-effect model described above was performed after 36 participants had completed all treatment periods. Estimates 


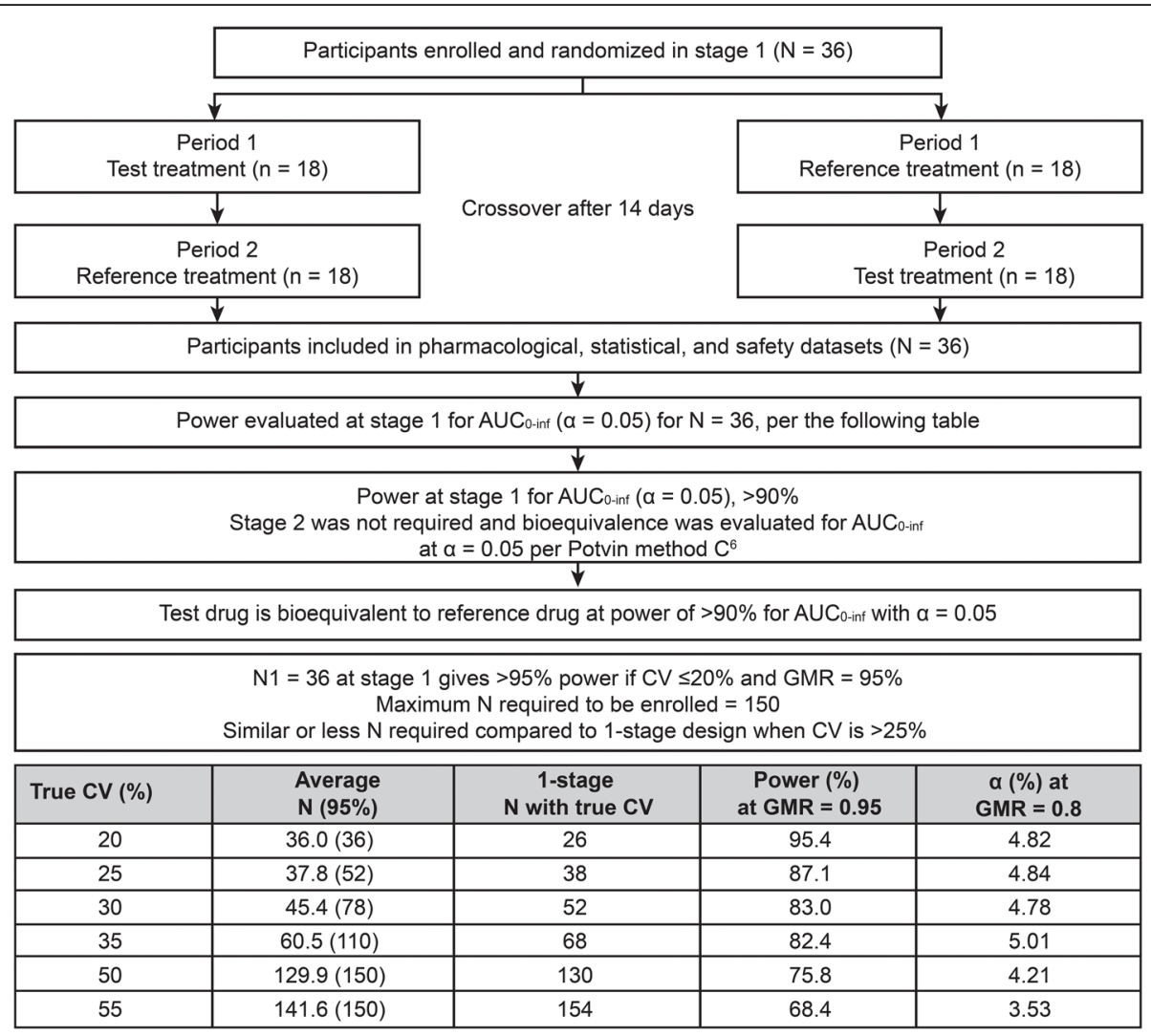

Fig. 1 Study design and disposition of participants. $A \cup C_{\text {o-inf }}$ area under the analyte concentration versus time curve from time zero to infinity, $C V$ coefficient of variation, GMR geometric mean ratio

of the within-participant $\mathrm{CV}$ for $\mathrm{AUC}_{0 \text {-inf }}$ were obtained. Power was estimated using the variance estimate from cohort 1 , assuming $\alpha$ level of 0.05 and a fixed true GMR of 0.95 using the same formula provided by Potvin et al. (Potvin et al. 2008). Health Canada, the agency that this study was filing to, only requires $90 \% \mathrm{CI}$ of $\mathrm{GMR}$ for $\mathrm{AUC}_{0 \text {-inf }}$ being within 0.80 and 1.25 . Thus, only the power for $\mathrm{AUC}_{0 \text {-inf }}$ was considered for decision making. However, the power requirement to trigger $\mathrm{BE}$ testing in stage 1 was raised from $80 \%$ to $90 \%$, so that it would maintain similar power requirement if both $\mathrm{AUC}_{0 \text {-inf }}$ and $\mathrm{C}_{\max }$ were considered assuming independence between the two PK endpoints. If the power was $\geq 90 \%$, a $90 \%(\alpha=0.05) \mathrm{CI}$ for $\mathrm{AUC}_{0 \text {-inf }}$ true GMR and point estimate for $\mathrm{C}_{\max }$ true GMR were calculated using the aforementioned model. Criteria for evaluation were based on results of an interim analysis from stage 1 . As the power was $\geq 90 \%$, stage 2 was not required regardless of the outcome of the bioequivalence evaluation, and the study was considered complete with the participants from stage 1 only.

All analyses were performed using $\mathrm{SAS}^{\bullet}$ software (version 9.2, SAS Institute Inc., Cary, NC) and R software version 3.0.2.

\section{Results}

\section{Demographic and baseline characteristics}

In total, 36 participants were enrolled and all completed stage 1 of the study. The mean (standard deviation [SD]) age of participants was $41(10)$ years, and $52.8 \%(n=19)$ were women (Table 1).

\section{Power estimation at stage 1}

At the interim analysis, the estimated power was > 99\% for $\mathrm{AUC}_{0 \text {-inf }}$ and the corresponding pseudo intraparticipant $\mathrm{CV}$ was $8.4 \%$. Therefore, stage 2 was not needed, the study was considered completed, and the statistical analysis was conducted with $\alpha=0.05$. There were no identified outliers (studentized residuals) and no predose concentrations $>5 \%$ of the corresponding $\mathrm{C}_{\max }$; therefore, the pharmacokinetic, statistical, and safety datasets included all 36 participants from cohort 1.

\section{Pharmacokinetic results}

The mean plasma diazoxide concentration-time profiles for the test and reference products are shown in Fig. 2. At the end of stage 1, a significant treatment effect was detected by the ANOVA for AUC from time zero to 
Table 1 Demographic and baseline characteristics of participants (safety, pharmacokinetic, and statistical datasets)

\begin{tabular}{ll}
\hline & $N=36$ \\
\hline Age (years), mean \pm SD & $41 \pm 10$ \\
Age group, n (\%) & $17(47.2)$ \\
18-40 years & $19(52.8)$ \\
41-64 years & \\
Sex, $\mathrm{n}(\%)$ & $17(47.2)$ \\
$\quad$ Male & $19(52.8)$ \\
Female & \\
Race, $\mathrm{n}(\%)$ & $10(27.8)$ \\
Asian & $8(22.2)$ \\
Black & $18(50.0)$ \\
White & \\
Ethnicity, $\mathrm{n}(\%)$ & $3(8.3)$ \\
Hispanic/Latino & $25.6 \pm 2.4$ \\
BMl (kg/m²), mean \pm SD & $168.1 \pm 8.7$ \\
Height (cm), mean \pm SD & $72.7 \pm 11.5$ \\
Weight (kg), mean \pm SD &
\end{tabular}

$B M I$ body mass index, $S D$ standard deviation

time of the measurable analyte concentration $\left(\mathrm{AUC}_{0-\mathrm{t}}\right.$; $p \leq 0.0001), \operatorname{AUC}_{0 \text {-inf }}(p=0.0052)$, and $C_{\text {max }}(p \leq 0.0001)$. ANOVA also detected a significant sequence effect in the analysis of $C_{\max }(p=0.0227)$. The GMs of $\mathrm{AUC}_{0-\mathrm{t}}, \mathrm{AUC}_{0-}$ inf, and $C_{\max }$ for test and reference products were 200,000 and 185,000 ng.hour/mL; 244,000 and 230,000 ng.hour/ $\mathrm{mL}$; and 5,830 and 4,990 ng/mL, respectively (Table 2).

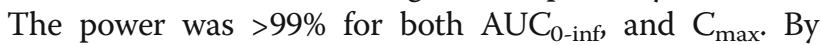
statistical comparison, the GMRs $(90 \% \mathrm{CI})$ of $\mathrm{AUC}_{0-\mathrm{t}}$, $\mathrm{AUC}_{0 \text {-inf }}$, and $\mathrm{C}_{\max }$ of test versus reference product were 1.08 (1.05-1.11), 1.06 (1.03-1.10), and 1.17 (1.14-1.20), respectively (Table 2 ). Thus, the study showed that bioequivalence criteria were met for the two products. The $90 \%$ CIs of GMR of $\mathrm{AUC}_{0 \text {-inf }}$ and $\mathrm{C}_{\max }$ were between 0.80 and 1.25 , proving the hypothesis that a single dose of diazoxide (100 mg capsule) manufactured in Patheon, France (test product) is bioequivalent to a single dose of diazoxide (100 mg capsule) manufactured in Comazzo, Italy (reference product).

The median (range) of $\mathrm{T}_{\max }$ for the test and reference products was $4.0(1.0-18.0)$ and $4.0(2.0-9.0)$ hours, respectively. The GM (\% CV) of the $t_{1 / 2}$ for the test and reference products was 27.5 hours (27.3) and 27.0 hours (31.3), respectively. The median (range) of $t_{1 / 2}$ for the test and reference products was $27.4(14.6-46.8)$ and 26.1 (15.9-51.5), respectively.

\section{Safety}

Both study products were generally well tolerated. Overall, 20 treatment-emergent adverse events (TEAEs) involving 9 participants were reported. No serious AEs were reported, and all AEs were mild in severity, with the most frequently reported $\mathrm{AE}$ being headache $(3 / 36$ [8.3\%] participants taking the reference product; Table 3). One participant experienced hyperglycemia $(11.9 \mathrm{mmol} /$ L) during period 1 (test product), and another experienced hypoglycemia $(3.2 \mathrm{mmol} / \mathrm{L})$ during period 2 (test product). Both AEs were mild and resolved without concomitant treatment and were considered possibly related to study drug by the investigators. One NTEAE of somnolence, which was also mild in severity, was reported (Table 3). One participant reported influenzalike symptoms during period 2 (test product).

\section{Discussion}

This pivotal bioequivalence study evaluated the relative bioavailability of a single dose of diazoxide $(100 \mathrm{mg})$, manufactured at two international sites (France and Italy), in healthy participants under fasting conditions using an adaptive, two-stage sequential design approach (Potvin et al. 2008). The study was considered complete after stage 1 because the power of the study was $>90 \%$ for $\mathrm{AUC}_{0 \text {-inf }}$ with $\alpha=0.05$. When compared with the reference product, the pharmacokinetic parameters of the test product were on average $8 \%, 6 \%$, and $17 \%$ higher

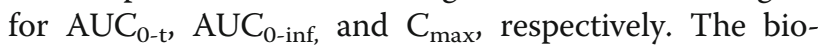
equivalence criteria were met as the $90 \% \mathrm{CI}$ of the GMR of $\mathrm{AUC}_{0 \text {-inf }}(1.06[1.03,1.10])$ and the point estimate $\mathrm{C}_{\max }(1.17[1.14,1.20])$ of the test versus reference product were within the range 0.80 to 1.25 . Therefore, the hypothesis that a single dose of diazoxide $(100 \mathrm{mg}$ capsule) manufactured in Patheon, France (test product) is bioequivalent to a single dose of diazoxide $(100 \mathrm{mg}$ capsule) manufactured in Comazzo, Italy (reference product) was supported.

In the present study, the standards set by the Health Canada guidance document for single-dose crossover comparative bioavailability studies were met on log-transformed parameters calculated from the measured data (Health Canada 2012). Also, the measured contents of the test and reference products used in the study (expressed as percentages of the label claim) were within $5 \%$ of each other per the FDA recommendations (FDA 2013).

In the absence of estimates of $\mathrm{CV}$ and GMR from published literature or pilot studies, the FDA recommends the sequential design approach as an alternative (FDA 2013). This approach uses an initial limited number of participants (planned $\mathrm{N}$ ) to evaluate bioequivalence. If necessary, more participants are enrolled (calculated $\mathrm{N}$ ) before a final bioequivalence evaluation. However, the challenge is to control type I error rate, $\alpha$ (participant's risk and the chance of approving a bioinequivalent product) for such approaches (Fuglsang 2013). 

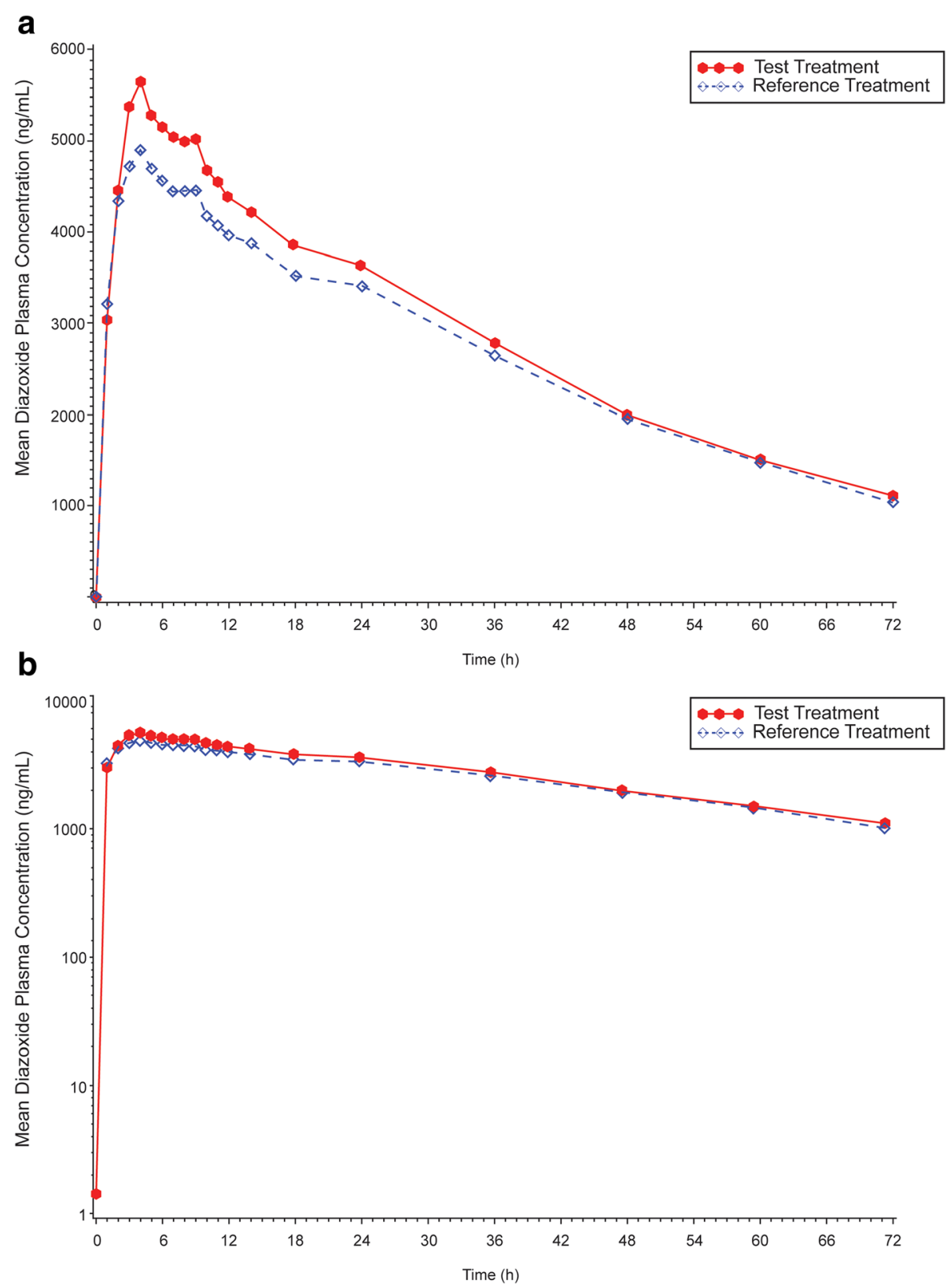

Fig. 2 Mean diazoxide plasma concentration-time profiles for the test and reference products a linear scale, $\mathbf{b}$ log-linear scale. Test, PROGLICEM ${ }^{\circledast}$ 100 mg hard capsules, Batch No.: 3107A (Merck Sharp \& Dohme Corp, USA); reference, PROGLYCEM ${ }^{\circledast} 100$ mg capsules, Lot No.: 120118 (Merck Canada Inc., Canada)

Group sequential approaches for bioequivalence studies have several advantages. These methods allow early discontinuation of the trial and provide the advantage of optimizing resource use in situations when the planned total sample size is based on an overestimated CV. However, no provision is made for changing the maximum number of participants, and protection against the study being overpowered or underpowered due to a poorly anticipated CV value is not guaranteed (Polli et al. 2012). Re-estimation approaches allow the final sample size to be estimated based on the CV estimate after stage 1 . An adaptive, two-stage sequential design has the advantage of both group sequential and re-estimation approaches for bioequivalence trials. An adaptive, two-stage sequential design also obviates the need for a "futility" criterion.

In pivotal simulation study sponsored by The Product Quality Research Institute, Potvin et al. compared four different two-stage methods for conducting bioequivalence trials; one was an internal pilot study design $(\operatorname{method} \mathrm{A})$ and the other three (methods $\mathrm{B}, \mathrm{C}$, and $\mathrm{D})$ 
Table 2 Pharmacokinetic results based on measured plasma study drug concentrations $(N=36)^{a}$

\begin{tabular}{|c|c|c|c|c|c|c|}
\hline Parameter & Product & GM & $\mathrm{GM}, 95 \% \mathrm{Cl}$ & GMR (Test vs Reference), \% & GMR, 90\% Cl & Pseudo Intra-participant \% CV \\
\hline \multirow[t]{2}{*}{$A \cup C_{0-t}$, ng.hour $/ \mathrm{mL}$} & Test & 200,000 & $184,000-217,000$ & 108.01 & $105.08-111.03$ & 6.9 \\
\hline & Reference & 185,000 & $170,000-201,000$ & - & - & - \\
\hline \multirow[t]{2}{*}{$\mathrm{AUC}_{0-\text { inf, }}$ ng.hour$/ \mathrm{mL}$} & Test & 244,000 & $219,000-272,000$ & 106.12 & $102.62-109.75$ & 8.4 \\
\hline & Reference & 230,000 & $206,000-257,000$ & - & - & - \\
\hline \multirow[t]{2}{*}{$C_{\max }, n g / m L$} & Test & 5,830 & $5,450-6,230$ & 116.89 & $113.66-120.22$ & 7.0 \\
\hline & Reference & 4,990 & $4,680-5,320$ & - & - & - \\
\hline
\end{tabular}

$A \cup C_{0 \text {-inf }}$ area under the curve from time zero to infinity, $A \cup C_{0-t}$ area under the curve from time zero to the time of the last measurable analyte concentration, $C_{m a x}$ maximum plasma analyte concentration, $C l$ confidence interval, $C V$ coefficient of variation, GM geometric mean, GMR geometric mean ratio

${ }^{a} A$ nonzero predose concentration level was obtained at the beginning of period 2 in one participant. However, the measured predose level was $<1 \%$ of the participant's corresponding $C_{\max }$ value. In compliance with the protocol, the predose level was maintained in the pharmacokinetic analysis without baseline correction

${ }^{b}$ Estimated based on the elements of the variance-covariance matrix as: $C V(\%)=100 * \operatorname{sqrt}\left[\left(\sigma \mathrm{A}^{2}+\sigma \mathrm{B}^{2}-2^{*} \sigma A B\right) / 2\right]$, where $A$ and $B$ are the two treatments Test, PROGLICEM ${ }^{\circledast} 100$ mg hard capsules, Batch No.: 3107A (Merck Sharp \& Dohme Corp, USA); reference, PROGLYCEM ${ }^{\circledR} 100$ mg capsules, Lot No.: 120118 (Merck Canada Inc., Canada)

were adaptive sequential methods (Potvin et al. 2008). These methods are based on a combination of group sequential and re-estimation approaches for bioequivalence trials. The simulation study recommended method $\mathrm{C}$ based on a small advantage in power and the fact that, if the study was found to have adequate power at stage 1 , the type I error rate for that study would be controlled around $5 \%$ and similar to that for a single-stage study

Table 3 Frequency of adverse events and nontreatment-emergent adverse events by preferred term

\begin{tabular}{lll}
\hline & AEs, $\mathrm{n}$ & \\
\hline Preferred Term & Test Treatment & Reference Treatment \\
Bacterial test positive & 1 & 1 \\
Dry throat & 1 & \\
Hyperglycemia & 1 & \\
Hypoglycemia & 1 & \\
Somnolence & 1 & \\
Urine leukocyte esterase positive & 1 & \\
Vomiting & 1 & \\
White blood cells urine positive & 1 & 1 \\
Dizziness & & 3 \\
Headache & & 1 \\
Influenza-like illness & & 1 \\
Nausea & & 1 \\
Nitrite urine present & 1 \\
Oropharyngeal pain & 1 \\
Sinus congestion & 1 \\
Urine leukocyte esterase positive & \\
White blood cells urine positive & \\
Total & & 1 \\
\hline NiEAE & & 1 \\
\hline
\end{tabular}

\section{${ }^{a} \mathrm{NTEAE}$}

AEs adverse events, NTEAE nontreatment emergent adverse event Test, PROGLICEM ${ }^{\ominus} 100 \mathrm{mg}$ hard capsules, Batch No.: 3107A (Merck Sharp \& Dohme Corp, USA); reference, PROGLYCEM ${ }^{\star} 100$ mg capsules, Lot No.: 120118 (Merck Canada Inc., Canada) design (Potvin et al. 2008). This method is also recommended by Health Canada when CV is unknown.

Given the lack of evidence suggesting high withinparticipant variation or GMR being different from 100\%, we concluded that Potvin's method $\mathrm{C}$ was a reasonable approach in the present scenario. The two-stage sequential design has the advantage of generating not more than minimal inflation of type I error rate (Potvin et al. 2008). In contrast to the single-stage design which needs recruitment of all participants at the same time, the smaller sample size recruited at stage 1 is a major advantage of this method. The bioequivalence criterion with $\alpha$ $=0.05$ corresponds to the conventional bioequivalence criteria of the $90 \%$ two-sided CI for the ratio of GMs falling within the 0.80 to 1.25 range. Results showed that the present study had adequate power ( $>90 \%$ ) at stage 1 , and the specified $\alpha=0.05$ for this study was similar to a single-stage study design. In the current study, the test product was found to be bioequivalent to the reference product at stage 1 with a sample of 36 participants.

In the present study, the significant sequence effect in the analysis of $\mathrm{C}_{\max }$ was considered a random event, given the single-dose design, involvement of healthy volunteers, sufficiently long washout period, predose levels from the second period being $<1 \%$ of the corresponding $\mathrm{C}_{\max }$, validated assay procedure, and diazoxide being an exogenous entity. The significant differences in treatment effects for $\mathrm{AUC}_{0-\mathrm{t}}(8 \%), \mathrm{AUC}_{0 \text {-inf }}(6 \%)$, and $C_{\max }(17 \%)$ were believed to have no clinical importance since the $90 \%$ CIs of the test to reference ratios were entirely within the 0.80 to 1.25 bioequivalence range for all three parameters.

There are two modifications in the present study from Potvin's method $C$ to ensure the success of the present study. First, 90\% power was chosen to ensure adequate power when evaluating bioequivalence at the interim and final analysis. Second, the choice of sample size made in the first stage $\left(n_{1}=36\right)$ is also critical to the 
success of study, and was not clearly recommended in Potvin's method C. Given no prior information on AUC or $\mathrm{C}_{\max }$ for diazoxide, the sample size at stage 1 was chosen to ensure adequate power ( 90\%) for intraparticipant $\mathrm{CV} \leq 25 \%$, a typical variability range observed for clinical pharmacokinetic parameters of most drug products.

Both study products were generally well tolerated. No serious AEs were reported, and all AEs, including TEAEs, were mild in severity. Safety and tolerability of the study products were in line with known side effects of diazoxide.

\section{Conclusion}

In an adaptive, two-stage, sequential design clinical bioequivalence study, a single dose of diazoxide (100 mg capsule) manufactured in Patheon, France (test product) was bioequivalent to a single dose of diazoxide $(100 \mathrm{mg}$ capsule) manufactured in Comazzo, Italy (reference product) in healthy normal subjects. An $\mathrm{N}$ of 36 participants

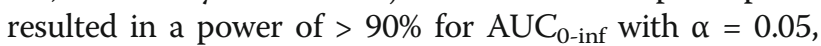
resembling a conventional, single-stage study design.

\begin{abstract}
Abbreviations
AEs: adverse events; ANOVA: analysis of variance; AUC: area under the plasma concentration time curve; $A \cup C_{0 \text {-inf. }} A \cup C$ from time zero to infinity; $A \cup C_{0-t}: A \cup C$ from time zero to time of the last measurable analyte concentration; BLQ: below the limit of quantitation; Cl: confidence interval; $C_{\text {max }}$ : peak plasma concentration; $\mathrm{CV}$ : coefficient of variation; FDA: Food and Drug Administration; GLP: good laboratory practice; GMR: geometric mean ratio; $\mathrm{K}_{\mathrm{e}}$ : apparent first-order elimination rate constant; LLOQ: lower limit of quantitation; NTEAEs: non-treatment-emergent adverse events; OECD: Organisation for Economic Co-operation and Development; SD: standard deviation; $t_{1 / 2}$ : apparent elimination half-life; TEAEs: treatmentemergent adverse events; $T_{\max }$ : time to $C_{\max }$
\end{abstract}

\section{Acknowledgements}

Medical writing and/or editorial assistance was provided by Vidula Bhole, MD, MHSc, of Cactus Communications, Mumbai. This assistance was funded by Merck \& Co., Inc., Kenilworth, NJ.

\section{Funding}

Funding for this study was provided by Merck \& Co., Inc., Kenilworth, NJ. The funder was inolving in the design of the study, collection, analysis, and interpretation of data and, writing of the manuscript.

\section{Authors' contributions}

MOB contributed to the conception, design, and planning of the study; acquisition and analysis of the data; interpretation of the results; drafting of the manuscript; and critical review or revision of the manuscript for important intellectual content. JX contributed to the conception, design, and planning of the study; analysis of the data; interpretation of the results; drafting of the manuscript; and critical review or revision of the manuscript for important intellectual content. DP contributed to the conception, design, and planning of the study; interpretation of the results; and critical review or revision of the manuscript for important intellectual content. PF contributed to the conception, design, and planning of the study; and critical review or revision of the manuscript for important intellectual content. All authors approved the final version for publication.

\section{Publisher's Note}

Springer Nature remains neutral with regard to jurisdictional claims in published maps and institutional affiliations.

Received: 12 January 2017 Accepted: 1 April 2017

Published online: 05 May 2017

\section{References}

Davit BM, Conner DP, Fabian-Fritsch B, Haidar SH, Jiang X, Patel DT, Seo PR, Suh K, Thompson CL, Yu LX (2008) Highly variable drugs: observations from bioequivalence data submitted to the FDA for new generic drug applications. AAPS J 10:148-156

Dayton PG, Pruitt AW, Faraj BA, Israili ZH (1975) Metabolism and disposition of diazoxide. A mini-review. Drug Metab Dispos 3:226-229

FDA (2010) Guidance for Industry: Adaptive Design Clinical Trials for Drugs and Biologics. https://www.fda.gov/downloads/Drugs/\%E2\%80\%A6/Guidances/ ucm201790.pdf. Accessed 15 Sept 2016.

FDA (2013) Guidance for Industry: Bioequivalence Studies with Pharmacokinetic Endpoints for Drugs Submitted Under an ANDA (DRAFT GUIDANCE). http:// www.fda.gov/downloads/drugs/guidancecomplianceregulatoryinformation/ guidances/ucm377465.pdf. Accessed 15 Sept 2016

Fenta HM (2014) Determination of sample size for two stage sequential designs in bioequivalence studies under $2 \times 2$ crossover design. Science Journal of Clinical Medicine 3:82-90

Fuglsang A (2013) Sequential bioequivalence trial designs with increased power and controlled type I error rates. AAPS J 15:659-661

Gribble FM, Tucker SJ, Ashcroft FM (1997) The essential role of the Walker A motifs of SUR1 in K-ATP channel activation by Mg-ADP and diazoxide. EMBO J 16:1145-1152

Health Canada (2012) Conduct and Analysis of Comparative Bioavailability Studies and Comparative Bioavailability Standards: Formulations used for Systemic Effects. http://www.hc-sc.gc.ca/dhp-mps/alt_formats/pdf/ prodpharma/applic-demande/guide-ld/bio/gd_cbs_ebc_ld-eng.pdf. Accessed 15 Sept 2016

Kenward MG, Roger JH (1997) Small sample inference for fixed effects from restricted maximum likelihood. Biometrics 53:983-997

O'Brien PC, Fleming TR (1979) A multiple testing procedure for clinical trials. Biometrics 35:549-556

Organisation for Economic Co-operation and Development (OECD) (2006) Good Laboratory Practice: OECD Principles and Guidance for Compliance Monitoring. OECD Publishing, France

Pocock SJ (1982) Interim analyses for randomized clinical trials: the group sequential approach. Biometrics 38:153-162

Polli JE, Cook JA, Davit BM, Dickinson PA, Argenti D, Barbour N, Garcia-Arieta A, Geoffroy JM, Hartauer K, Li S, Mitra A, Muller FX, Purohit V, Sanchez-Felix M, Skoug JW, Tang K (2012) Summary workshop report: Facilitating oral product development and reducing regulatory burden through novel approaches to assess bioavailability/bioequivalence. AAPS J 14:627-638

Potvin D, DiLiberti CE, Hauck WW, Parr AF, Schuirmann DJ, Smith RA (2008) Sequential design approaches for bioequivalence studies with crossover designs. Pharm Stat 7:245-262

PROGLYCEM (2015) Product Monograph., http://www.merck.ca/assets/en/pdf/ products/PROGLYCEM-PM_E.pdf. Accessed 15 Sept 2016

US Department of Health and Human Services. Good Laboratory Practice for Nonclinical Laboratory Studies. 21 CFRß58. Washington, DC: April 2010. https://www.fda.gov/ohrms/dockets/98fr/980335s1.PDF

Vaziri ND (1984) Malignant or accelerated hypertension. West J Med 140:575-582

\section{Disclosure statement}

All authors report other disclosures from Merck \& Co., outside the submitted work. 